\title{
Avaliação econômica da poluição do ar na Amazônia Ocidental: um estudo de caso do Estado do Acre $^{1}$
}

\author{
Rubicleis Gomes da Silva ${ }^{2}$ \\ João Eustáquio de Lima ${ }^{3}$
}

Resumo: Os problemas ocasionados pela poluição do ar em razão das queimadas existentes dentro e fora do Estado do Acre têm causado sérios prejuízos à saúde da população. Como as queimadas provocam malefícios à sociedade, a mensuração da disposta a pagar (DAP) para diminuir esses impactos, constitui importante informação para avaliar se um projeto de despoluição é viável economicamente. De forma geral, objetivou-se analisar os impactos da poluição do ar pelas queimadas sobre a sociedade acreana. Especificamente: a) determinar quanto a sociedade está disposta a pagar pela diminuição dos malefícios ocasionados pelas queimadas; e b) comparar o valor da disposição a pagar agregada com o custo das morbidades respiratórias no ano de 2004. A teoria das externalidades é utilizada para referenciar este trabalho. A partir de referendum with follow-up, estimou-se o valor dos benefícios da melhoria da qualidade do ar no Estado do Acre cujos resultados indicaram que cada dólar aplicado em despesas de internações ocasionadas por morbidades respiratórias à melhoria do ar acarreta um benefício de $\mathrm{R} \$ 21,08$, o que representa que a melhoria

\footnotetext{
${ }^{1}$ Os autores agradecem a Universidade Federal do Acre (UFAC) e a CAPES/PQI pelo financiamento da pesquisa, e aos pareceristas anônimos pelos comentários e sugestões. ${ }^{2}$ D.S. em Economia Aplicada e Professor Adjunto de Métodos Quantitativos do Departamento de Economia da UFAC. rubicleis@uol.com.br

${ }^{3}$ Ph.D em Economia Agrícola e Professor de Titular do Departamento de Economia Rural da Universidade Federal de Viçosa - UFV. jelima@ufv.br
} 
dessa característica ambiental é viável economicamente. Por fim, o valor máximo que a sociedade se dispõe a contribuir para a melhoria da qualidade do ar no Estado do Acre pode ser utilizado para o financiamento de projetos de desenvolvimento de tecnologia e extensão rural que possibilitem criar condições objetivas para o produtor rural incorporar em seu processo de produção agropecuária práticas alternativas às queimadas no preparo do solo.

Palavras-chave: Amazônia, poluição do ar, método de valoração contingente e Estado do Acre.

Classificação JEL: Q51, Q53, D62, C35

Abstract: The problems caused by air pollution as a function of burned-over lands in and out of Acre state cause grave injuries to the population health. Since the burned-over lands cause damages to society, the monetary value mensuration which the same is willing to pay to diminish their impacts constitutes important information to evaluate whether a depolluting project is economically feasible. In general, this research sought to examine the impacts that the ar pollution for fire vegetation has on the Acrean society. Specifically, a) to determine how much society is willing to pay for the decrease in the injuries caused by burned lands, and b) to compare the aggregated willingness-to-pay (WTP) value with the cost of the respiratory morbidity occasioned by the burned lands for the year 2004. The externalities theory was utilized to reference this work. The referendum with follow-up was utilized to estimate the benefit for depollution of air of the Acre State. The results show that the dollar applied in internments expenditures for respiratory morbidity aiming at air bettering brings a benefit of US\$21.08 meaning that the improvement of this environmental characteristic is economically feasible. At last, the value that society disposes to contribute for air improvement in State of Acre can be utilized to finance the development of technologies and rural extension projects and make possible to create objective conditions to the rural producer to incorporate in his productive process an alternative practice to burned lands in the soil prepare for the farming production process. 
Key words: Amazon, air pollution, contingent valuation method, Acre State.

JEL Classification: Q51, Q53, D62, C35

\section{Introdução}

O uso do fogo é uma prática comum e de baixo custo utilizada para formação de lavoura e pastagens na região Amazônica. Contudo, este tipo de prática agrícola, ocasiona graves prejuízos devido à poluição do ar à população das regiões onde são praticadas as queimadas, bem como, em outras regiões geográficas, dado o deslocamento da fumaça e fagulhas pelas correntes de ar.

Os problemas ocasionados pela poluição do ar, em decorrência das queimadas dentro e fora do Estado do Acre, causam sérios prejuízos ao bem-estar (saúde e comodidade) da sociedade acreana. Dado que as queimadas causam malefícios à sociedade, principalmente, à saúde dos cidadãos, a mensuração do valor monetário que esta estaria disposta a contribuir para diminuir seus impactos, constitui importante informação para determinação dos prejuízos causados à população, bem como, é um indicador que fornece um valor que pode ser utilizado como fonte de financiamento para o desenvolvimento de projetos que possibilitem a diminuição das queimadas no Estado do Acre e, consequentemente, seus efeitos negativos.

Nesse contexto, a mensuração da disposição a pagar (DAP) pela melhoria da qualidade do ar é uma informação relevante para os diversos níveis de tomada de decisão para formulação de políticas públicas e privadas que visem criar mecanismos para a diminuição dos impactos negativos ocasionados pela utilização do fogo no processo produtivo da agropecuária da região amazônica, e principalmente, do Estado do Acre.

Um dos principais impactos negativos ocasionados pela poluição do ar em virtude das queimadas no Acre, está na taxa de mortalidade associada às morbidades respiratórias, neste sentido, Silva (2005) destaca, que no período de 1998 a 2004, houve uma diferença de aproximadamente, $21 \%$ nesta taxa no período das queimadas e não-queimadas. No período das queimadas, a taxa de mortalidade foi de 3,3 por mil/hab, 
enquanto no da não-queimada, essa taxa foi de 2,73, Este dado ilustra, que a utilização do fogo como fator de produção agrícola na Amazônia, impacta negativamente, na saúde da sociedade local.

O que este estudo traz de novo nas aplicações da valoração contingente é a análise da poluição do ar ocasionada pelas queimadas na Amazônia, especificamente no Estado do Acre. Em se tratando desta região, este estudo possui grande relevância, pois elabora uma avaliação econômica da melhoria da qualidade dor ar.

De forma geral, esta pesquisa busca determinar quanto a sociedade acreana estaria disposta a contribuir para diminuição dos impactos negativos advindos das queimadas. Especificamente, busca-se: a) verificar quais variáveis sócio-econômicas possuem maior relevância para explicar a decisão do cidadão sobre se dispor a contribuir, ou não, pela melhoria da qualidade do ar no Acre; e b) comparar o valor da disposição a pagar agregada pela melhoria da qualidade do ar, com o custo das morbidades respiratórias no ano de 2004.

Este artigo contém além desta introdução, mais três seções. Na próxima, discute-se o referencial teórico utilizado e sua vinculação com o problema de pesquisa, em seguida, o referencial analítico é apresentado, posteriormente os resultados são apresentados e algumas discussões são realizadas; e por fim, são apresentadas as principais conclusões obtidas da análise dos resultados.

\section{Referencial teórico}

A atividade agropecuária na Amazônia e especificamente no Acre, utiliza com muita freqüência e intensidade, as queimadas como fator de produção em virtude de estas apresentarem baixo custo e a fiscalização dos órgãos competentes serem deficientes. Em razão de este "fator de produção" (queimadas) apresentar baixo custo, ele contribui pelo menos no curto prazo, para o aumento da produção agropecuária.

Dois aspectos merecem especial relevância: o primeiro é o do impacto positivo na produção agropecuária, e o segundo é o impacto negativo no bem-estar da população acreana, especificamente na saúde. Os impactos positivos sobre a produção agropecuária dão-se em função das queimadas reduzirem a curtíssimo prazo o custo de produção do 
produtor agrícola, pois a utilização do fogo como fator de produção é menos dispendiosa do que outras tecnologias disponíveis para preparo do solo e pastagens. O aspecto negativo ocorre em função da emissão de Carbono $\left(\mathrm{CO}_{2}\right)$ e outros poluentes na atmosfera, consequentemente aumentando a poluição do ar e causando impactos negativos sobre o bem-estar da população local.

A utilização do fogo como fator de produção torna a produção agrícola maior que o ótimo social pois o produtor não incorpora em seu processo de produção os impactos negativos que as queimadas ocasionam sobre a sociedade.

Uma externalidade surge quando a relação de produção ou utilidade de uma firma ou indivíduo inclui variáveis cujos valores são escolhidos por outros sem levar em conta o bem-estar do afetado, além disto, os causadores dos efeitos não pagam nem recebem nada pela sua atividade, este fato ressalta o caráter involuntário da externalidade.

O conceito apresentado acima possui sintonia direta com duas condições que Pearce e Turner (1990) destacaram para a existência de custos ou benefícios externos, as quais são:

- as atividades de um agente podem causar perdas ou ganhos de bem-estar em outros agentes; e

- as perdas e os ganhos de bem-estar não são compensados financeiramente.

No Estado do Acre o produtor em seu processo de produção agropecuária utiliza com bastante freqüência e intensidade as queimadas para a formação de lavouras e pastagens. O baixo custo da utilização desta "tecnologia suja”, ocasiona uma expansão da produção acima do nível sem a utilização das queimadas. Contundo, a utilização das queimadas causam externalidades negativas sobre a população acreana. Em função das externalidades não estarem gravadas no processo produtivo, a produção com utilização das queimadas é superior à produção com a gravação da externalidade negativa, logo, ocorre uma diferença entre o ótimo social (ponto "A") e o ótimo privado (ponto "B”), ambos na Figura 1. 
Figura 1 - Curvas de custos marginais sociais, privados e externos associados à produção agropecuária na Amazônia

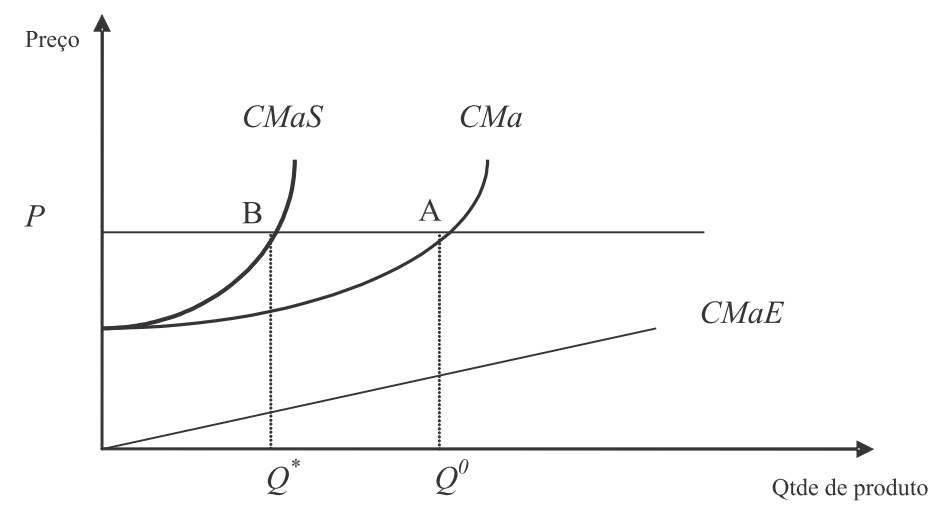

O ponto "A" é representado pela intersecção entre o custo marginal social (CMaS) e o preço de mercado $(P)$. O CMaS representa a soma do custo marginal da produção com o custo marginal da externalidade $(C M a E)$, ou seja, é a incorporação da externalidade a estrutura de custo do produtor, por sua vez, o custo marginal (CMa) representa apenas a variação do custo total em função da variação na quantidade produzida na atividade agropecuária, logo, não existe a internalização da externalidade. A partir disto, observa-se claramente, que no ponto “B”, onde a externalidade é incorporada a estrutura de custo da firma, a produção é inferior ao ponto “A”onde não existe a incorporação dos impactos negativos.

\section{Referencial analítico}

O método de valoração contingente (MVC) busca por meio de surveys (entrevistas) pessoais, revelar as preferências dos indivíduos por um bem ou serviço ambiental, ou seja, busca captar a disposição a pagar para garantir um benefício ou a disposição a aceitar para incorrer em um malefício. O MVC estima um valor monetário que reflete as preferências dos consumidores, relativas ao acréscimo/decréscimo na qualidade de ativos ambientais, consequentemente sobre seu bem-estar, o que nesta pesquisa, é representado pela melhoria da qualidade do ar. 


\subsection{Referendum with follow-up}

O referendum with follow-up (RF) foi primeiramente proposto por Carson et al. (1986), e Hanemann et al. (1991) fizeram a demonstração teorica e empírica de que os estimadores do RF são mais eficientes do que os estimadores do referendum single proposto por Hanemann (1984). No RF para cada entrevistado dois lances são apresentados, sendo o nível do segundo lance contingente ao primeiro. Se o indivíduo responder "sim" ao primeiro lance $\left(B_{i}\right)$, o segundo lance $\left(B_{i}^{u}\right)$ deverá ser maior que o primeiro $\left(B_{i}<B_{i}^{u}\right)$; caso o entrevistado responda "não" ao primeiro lance, o segundo lance $\left(B_{i}^{d}\right)$ deverá ser menor que o primeiro $\left(B_{i}^{d}<B_{i}\right)$.

Logo, quatro resultados são possíveis de ocorrer: a) ambas as respostas serem afirmativas; b) ambas as respostas serem negativas; c) a primeira resposta é negativa, seguida de uma resposta afirmativa; e d) a primeira resposta é afirmativa, seguida de uma resposta negativa. Esses quatro resultados são denotados, respectivamente, por $\pi^{s s}, \pi^{n n}, \pi^{n s}, \pi^{s n}$.

Quanto maior for o lance oferecido ao entrevistado menor será a probabilidade dele se dispor a contribuir. No processo de entrevista é oferecido o primeiro lance ao indivíduo $B_{i}$; caso ele rejeite este lance, é oferecido um lance menor $B_{i}^{d}$. Neste processo, o aumento do lance, diminui, respectivamente, a probabilidade de o "consumidor" se dispor a contribuir pela melhoria da qualidade do ar no Estado do Acre.

$\mathrm{O}$ valor que o entrevistado aceita a contribuir pela melhoria da qualidade do ar representa o valor máximo que o consumidor estará disposto a pagar. Neste contexto e utilizando a suposição de maximização de utilidade, tem-se as seguintes funções de verossimilhança:

No caso que $B_{i}^{u}>B_{i}$, ou seja, um "sim" seguido de um "sim", tem-se:

$$
\begin{aligned}
& \pi^{s s}=\left(B_{i}, B_{i}^{u}\right)=\operatorname{Pr}\left\{B_{i} \leq \max D A P \text { e } B_{i}^{u} \leq \max D A P\right\} \\
& \pi^{s s}=\left(B_{i}, B_{i}^{d}\right)=1-G\left(B_{i}^{u} ; \theta\right)
\end{aligned}
$$

No caso que $B_{i}>B_{i}^{d}$, ou seja, um "não" seguido de um "não", tem-se:

$$
\begin{aligned}
& \pi^{n n}=\left(B_{i}, B_{i}{ }^{d}\right)=\operatorname{Pr}\left\{B_{i}>\max D A P \text { e } B_{i}^{d}>\max D A P\right\} \\
& \pi^{n n}=\left(B_{i}, B_{i}^{u}\right)=G\left(B_{i}^{d} ; \theta\right) .
\end{aligned}
$$


Quando um "não" for seguido de um “sim”, tem-se, $\mathrm{B}_{i}^{d}<\mathrm{B}_{i}$, que é representado por:

$$
\begin{aligned}
& \pi^{n s}=\left(B_{i}, B_{i}^{d}\right)=\operatorname{Pr}\left\{B_{i} \geq \max D A P \geq B_{i}^{d}\right. \\
& \pi^{n s}=\left(B_{i}, B_{i}^{d}\right)=G\left(B_{i} ; \theta\right)-G\left(B_{i}^{d} ; \theta\right) .
\end{aligned}
$$

Por fim, quando um "sim” for seguido por um “não”, tem-se $\mathrm{B}_{i}^{u}>$ $\mathrm{B}_{i}$, que é representado por:

$$
\begin{aligned}
& \pi^{s n}=\left(B_{i}, B_{i}^{u}\right)=\operatorname{Pr}\left\{B_{i} \leq \max D A P \leq B_{i}^{u}\right. \\
& \pi^{s n}=\left(B_{i}, B_{i}^{u}\right)=G\left(B_{i}^{u}, \theta\right)-G\left(B_{i} ; \theta\right)
\end{aligned}
$$

Dado uma amostra de $\mathrm{N}$ entrevistados, em que $B_{i}$, $B_{i}^{u}$ e $B_{i}^{d}$ são lances oferecidos para o ísimo respondente, a função log-verossimilhança tem a seguinte forma:

$$
\ln L^{D}(\theta)=\sum_{i=1}^{N}\left\{\begin{array}{l}
d_{i}^{s s} \ln \pi^{s s}\left(B_{i}, B_{i}^{u}\right)+d_{i}^{n n} \ln \pi^{n n}\left(B_{i}, B_{i}^{d}\right)+ \\
d_{i}^{s n} \ln \pi^{s n}\left(B_{i}, B_{i}^{u}\right)+d_{i}^{n s} \ln \pi^{n s}\left(B_{i}, B_{i}^{d}\right)
\end{array}\right.
$$

Observe que as variáveis $d_{i}^{\text {yy }}, d_{i}^{\text {nn }}, d_{i}^{\text {ny }}$ e $d_{i}^{\text {yn }}$ são variáveis dicotômicas e correspondem às respostas dos entrevistados. Os estimadores de máxima verossimilhança, para o referendum with follow-up, são os valores do vetor de parâmetros $\theta$ que maximiza (5) e podem também ser obtidos pela solução do seguinte sistema de equações ${ }^{4}$ :

$$
\frac{\partial \ln L^{D}\left(\hat{\theta}^{D}\right)}{\partial \hat{\theta}}=0
$$

A matriz de variância e covariância dos parâmetros estimados é dada por:

$$
V^{D}\left(\hat{\theta}^{D}\right)=\left[-E \frac{\partial^{2} \mathrm{~h} L^{D}\left(\hat{\theta}^{D}\right)}{\partial \theta \partial \theta^{\prime}}\right]^{-1} \equiv I^{D}\left(\hat{\theta}^{\hat{D}}\right)^{-1}
$$

${ }^{4}$ Para maximização desta função foi construído um algoritmo no Limdep 8.0, o mesmo pode ser obtido com os autores. 
Na maximização da equação (5) é necessário observar que não existe qualquer relação entre o primeiro e o segundo lance. A maximização de (5) foi feita utilizando a função logística.

\subsection{Determinação do desvio-padrão da DAP pela melhoria da qualidade do ar}

O experimento de Krinsk-Roob $(1986)^{5}$ surgiu como alternativa a metodologia tradicional para calcular os desvios-padrões das elasticidades de funções de custo em funções Translog e Leontief. O objetivo da utilização deste experimento neste trabalho é determinar o desvio-padrão associado à DAP pela melhoria da qualidade do ar no Estado do Acre.

A operacionalização do método de Krinsk-Roob (1986) é simples. Por meio de uma distribuição normal multivariada com média e variância dos parâmetros do modelo estimado, geram-se $n$ amostras aleatórias pela utilização de simulação do Monte Carlo. De forma geral, os passos para elaboração do experimento de Krinsk-Roob são os seguintes:

1. Estimar o modelo desejado e identificar os parâmetros que serão utilizados na simulação;

2. Determinar a quantidade de replicações desejadas;

3. Simular o parâmetro desejado por meio de experimento de Monte Carlo, utilizando-se da média e da variância dos parâmetros estimados; e

4. Computar o resultado de cada simulação.

\subsection{Fonte de dados}

Os dados utilizados neste trabalho são procedentes de fonte primária coletada mediante entrevista em bairro da capital do Estado do Acre, Rio Branco, na terceira semana do mês de novembro de 2004. A escolha de Rio Branco como alvo da amostra é justificada por três motivos: o primeiro, pelo tamanho da população da capital em relação ao Estado. Conforme aponta o Instituto Brasileiro de Geografia e Estatística (IBGE),

${ }^{5}$ Para operacionalização deste procedimento foi construído um programa no Eviews 4.1, o mesmo pode ser obtido com os autores 
Rio Branco possui aproximadamente, $45,85 \%$ da população do Acre, o que corresponde a 284.555; o segundo, pelos aspectos financeiros, pois realizar pesquisa em um município é menos dispendioso do que em vários; terceiro, por Rio Branco possuir o maior número de focos de queimadas do Estado e, além disso, possui o maior número de morbidades respiratórias.

O tamanho da amostra foi estimado pela expressão (8), para amostras estatisticamente infinitas (acima de 100.000 observações). A amostra depende da percentagem com que o fenômeno ocorre, de seu complemento, do erro máximo permitido e do nível de confiança escolhido. Nesse caso, o tamanho da amostra será dado por:

$$
\mathrm{n}=\frac{\sigma^{2} \cdot \mathrm{p} \cdot \mathrm{q}}{\mathrm{e}^{2}}
$$

em que $n$ é o tamanho da amostra; $\sigma^{2}$, nível de confiança escolhido, expresso em número de desvios-padrão; $p$, probabilidade de o fenômeno ocorrer; $q$, probabilidade complementar; $\mathrm{e}^{2}$, erro máximo permitido.

Para determinação da amostra desta pesquisa adotaram-se três desvios-padrões como nível de confiança. Para a probabilidade de ocorrência do evento "p", adotou-se 0,50 , em razão do não-conhecimento da ocorrência deste evento, conseqüentemente, “q" é igual a 0,50, e o erro máximo "e" permitido é de $10 \%$. Como forma de minimizar os possíveis problemas nas respostas dos questionários, acresceu-se um percentual de $10 \%$ sobre o número da amostra. A equação (8) resultou em uma amostra de 225 indivíduos; no entanto, ao serem adicionados $10 \%$ na margem de segurança, obtiveram-se 248 entrevistados.

O procedimento metodológico utilizado nas entrevistas foi primeiro a determinação da quantidade de bairros que seriam pesquisados em cada região administrativa de Rio Branco, para este caso, foram escolhidos intencionalmente dois bairros por região, sendo que, ao todo, dez bairros foram pesquisados.

Em relação aos bairros pesquisados, estes foram determinados por meio de sorteio, tornando, assim, o procedimento aleatório. Foram entrevistados os chefes familiares das residências nas ruas principais dos bairros, e a justificativa para a não adoção de procedimento aleatório para esta fase da pesquisa foi a segurança dos entrevistadores, pois, 
no caso de sorteios das ruas, haveria a possibilidades de ruas com alto índice de violência serem sorteadas.

Após a determinação das ruas, o procedimento que foi utilizado na determinação das residências que foram entrevistadas foi o seguinte: no lado direito, foi entrevistada uma residência e outra não, assim sucessivamente, até o fim da rua. Já no lado esquerdo da outra rua foi entrevistada a primeira residência e duas foram puladas, e assim sucessivamente.

Quatro estudantes universitários foram responsáveis pela entrevistas que foram realizadas nos períodos da manhã, tarde e noite. Por fim, a determinação do número de entrevistas em cada bairro/região foi feita de forma proporcional, ou seja, a região com maior número de habitantes teve o maior número de entrevistados.

Nesta pesquisa, em virtude da existência de conhecimento prévio sobre o objeto de pesquisa, bem como sobre o método de valoração contingente, optou-se pela determinação dos lances com base na experiência dos autores. A Tabela 1 mostra os valores dos lances que foram oferecidos aos entrevistados.

Tabela 1 - Lances oferecidos aos entrevistados, pela melhoria na qualidade do ar no Estado do Acre, Amazônia, Brasil, 2004 (em R\$)

\begin{tabular}{cccc}
\hline & Lance inicial (sortear) & Lance inferior & Lance superior \\
1 & 2,00 & 1,00 & 3,00 \\
2 & 4,00 & 2,00 & 6,00 \\
3 & 8,00 & 4,00 & 12,00 \\
4 & 16,00 & 8,00 & 24,00 \\
5 & 32,00 & 16,00 & 48,00 \\
\hline
\end{tabular}

Fonte: Elaborado pelos autores.

\section{Resultados e discussões}

\subsection{Análise das características sócio-econômicas dos entrevistados e sua percepção sobre os impactos negativos oriundos das queimadas}

Foram entrevistados 101 homens e 144 mulheres, que em termos relativos representam 41,20 e $58,80 \%$ do total dos entrevistados. Do total da amostra, 76,60\% provinham do primeiro distrito de Rio Branco - Acre, enquanto $24,40 \%$, do segundo distrito administrativo. 
Dentre os vários problemas ambientais que são relativamente comuns no Acre, $49 \%$ dos entrevistados destacaram que as queimadas era o problema que mais lhes preocupava, seguidas da poluição dos rios e igarapés, 29,80\% das preocupações e, posteriormente o desflorestamento, $14,30 \%$ e as demais opções representaram 6,90\%.

Os resultados demonstraram que as queimadas exercem grandes impactos na percepção ambiental dos entrevistados possivelmente em razão de estas terem grande impacto no bem-estar da sociedade. Ao serem perguntados se durante o período de junho a outubro (período de queimadas na Amazônia) sentem algum desconforto em decorrência das queimadas $86,50 \%$ dos entrevistados responderam que sim, e o restante $13,50 \%$, não. $\mathrm{O}$ fato que merece destaque é que grande parte dos entrevistados sentia algum desconforto no período de queima.

Questionados sobre a freqüência em que algum ente familiar foi acometido de alguma doença respiratória nos últimos dois anos, no período das queimadas, 99,60\% disseram que algum deles havia sofrido de algum problema respiratório, enquanto que $46,30 \%$ disseram havia tido mais de quatro casos de doenças respiratórias durante os dois anos anteriores. Esse resultado mostra, claramente, que as queimadas exercem externalidades negativas sobre o bem-estar da comunidade.

Aproximadamente $99 \%$ dos entrevistados tiveram casos de doenças respiratórias no período de dois anos, contudo, é necessário cautela na interpretação desse valor, pois, em um ano em determinada família, é possível que ninguém tenha sofrido de doença respiratória, no entanto, a partir do momento em que o período de tempo aumenta, esses casos de doenças respiratórias aumentam a probabilidade de incidência, sendo que das famílias que tiveram casos de morbidades respiratórias nos dois anos anteriores, 59,09\% dos casos deram-se em jovens e crianças, $29,55 \%$ em adultos e $11,36 \%$ em idosos. 


\subsection{Análise das respostas do referendum with follow-up ${ }^{6}$ e do modelo logit duplo}

O procedimento para análise das respostas relacionadas com os lances oferecidos aos entrevistados conduziu à elaboração da Tabela 2 . O primeiro aspecto a ser analisado na Tabela 2 reside na porcentagem de entrevistados que não se dispuseram a contribuir que representou 45,30\% do total da amostra. Em relação à discriminação dos lances, os entrevistados que aceitaram ambos os lances totalizaram 67 pessoas, o que representa $27,35 \%$ dos entrevistados; já os que aceitaram o primeiro lance e rejeitaram o segundo perfizeram $15,51 \%$, por sua vez, os entrevistados que rejeitaram o primeiro lance e aceitaram o segundo representaram 11,83\%.

Tabela 2 - Respostas do referendum with follow-up para melhoria da qualidade do ar no Estado do Acre, 2004

\begin{tabular}{crrr}
\hline \multirow{2}{*}{ Respostas } & \multicolumn{3}{c}{ Total } \\
& \multicolumn{1}{c}{ Não } & \multicolumn{1}{c}{ Sim } & \multicolumn{1}{c}{ Total } \\
\hline \multirow{2}{*}{ Não } & 111 & 29 & 140 \\
& $45,30 \%$ & $11,83 \%$ & $100,00 \%$ \\
Sim & 38 & 67 & 105 \\
& $15,51 \%$ & $27,35 \%$ & $100,00 \%$ \\
Total & 149 & 96 & 245 \\
& $60,80 \%$ & $39,20 \%$ & $100,00 \%$ \\
\hline
\end{tabular}

Fonte: Resultados da pesquisa.

A maximização da função de verossimilhança, dada por (5) conduziu à estimação do modelo logit duplo mostrado na Tabela 3.

${ }^{6} \mathrm{O}$ método referedum with follow-up foi escolhido em função deste ter apresentado o melhor desempenho em relação aos demais métodos analisados. Cinco métodos foram utilizados nesta pesquisa que são: Hanemann (1984), Cameron (1988), Hanemann et al. (1991), Cameron e Quiggin (1994), Cooper et al. (2002). Dois critérios foram utilizados para determinar o melhor modelo, que foram: menor coeficiente de variação da DAP obtidas por experimento de Krinsk-Roob e o nível de significância dos parâmetros estimados. Para maiores detalhes ver Silva (2005). 
Tabela 3 - Parâmetros estimados do modelo logit duplo para melhoria da qualidade do ar no Estado do Acre, 2004

\begin{tabular}{ccccccc}
\hline Variáveis & Coeficientes & $\begin{array}{c}\text { Erro } \\
\text { Padrão }\end{array}$ & t-student & P-valor & $\begin{array}{c}\text { Efeito } \\
\text { marginal }\end{array}$ & $\begin{array}{c}\text { Elasticidades de } \\
\text { probabilidade }\end{array}$ \\
\hline C & $0,2744^{\text {ns }}$ & 0,2544 & 1,079 & 0,2807 & - & - \\
DAP $^{7}$ & $-0,2551^{* * *}$ & 0,0168 & 15,215 & 0,0000 & $-5,9357$ & 1,4423 \\
YF & $0,0006^{* * *}$ & 0,0001 & 4,766 & 0,0000 & 0,0126 & 0,4401 \\
G & $0,0016^{* *}$ & 0,0006 & 2,354 & 0,0186 & 0,0353 & 0,1022 \\
QC & $0,16022^{*}$ & 0,0848 & 1,887 & 0,0591 & 3,4649 & 0,1314 \\
DDIST & $0,35411^{\text {ns }}$ & 0,3625 & 0.975 & 0,3295 & 7,6611 & 0,0484 \\
\hline
\end{tabular}

Fonte: Resultado da pesquisa.

*** significativo a $1 \%$; ** significativo a $5 \%$; * significativo a $10 \%$; e NS não-significativo.

YF - renda familiar mensal em reais; DAP - disposição mensal a pagar em reais; $\mathrm{G}$ - gasto anual com doenças respiratórias em reais; QC - quantidade de crianças na família; e DDIST - dummy geográfica, 0 primeiro distrito de Rio Branco e 1 segundo distrito. O número da amostra é de 245 entrevistados.

O primeiro aspecto a ser analisado no modelo estimado reside na análise dos sinais. Excluindo a DAP, os demais parâmetros estimados apresentaram sinais positivos, o que significa que se essas variáveis possuíssem variações positivas aumentaria a probabilidade de o "consumidor" se dispor a pagar, caso contrário, diminuiria a probabilidade. Por sua vez, o sinal negativo da DAP indica relação inversa entre a probabilidade do "consumidor" se dispor a contribuir e possíveis aumentos da DAP, ou seja, à medida que o valor do lance aumenta a probabilidade do indivíduo se dispor a contribuir para a melhoria da qualidade do ar diminui.

Em relação às medidas de qualidade de ajustamento do modelo, o índice de razão de verossimilhança (LRI), o popular $\mathrm{R}^{2}$ de McFadden, indicou que $54,40 \%$ das variações nas variáveis explicativas explicam as variações nas variáveis dicotômicas. Embora esse valor não seja alto, é extremamen-

${ }^{7}$ A pergunta utilizada para captar a DAP dos entrevistados foi a seguinte:

- O problema da poluição do ar em função das queimadas no Acre atinge toda a população acreana. Observa-se, claramente, que no período de julho a outubro, o mal estar causado pela poluição do ar, prejudica crianças e adultos, toda sociedade acreana sofre algum desconforto, aeroportos são fechados, o cheiro de material queimado é muito forte, irritação ocular atinge muitas pessoas, fagulhas sujam muitas residências e outros problemas são ocasionados. Dentro deste cenário, você aceitaria contribuir, mensalmente, $\mathrm{R} \$$ (sortear o valor "lance inicial") através de sua conta de luz por um período de um ano, para melhorar a qualidade do ar e diminuir os impactos negativos ocasionados pela poluição do ar? 
te significativo, visto que a maximização da função de verossimilhança do logit duplo é bem mais complicada do que a do logit convencional. O teste de LR (razão de verossimilhança) apresentou um valor de 342,96, o que indica que pelo menos um parâmetro estimado é diferente de zero, por fim, o valor assumido do pseudo $\mathrm{R}^{2}$ foi de 0,1393 . Contudo, essa medida não é um bom indicador para avaliação de modelos binários.

A probabilidade média de o indivíduo se dispor a contribuir é de $31,64 \%$, sendo que o efeito marginal da DAP indica que para acréscimo de $\mathrm{R} \$ 1,00$ no valor do lance, a probabilidade do indivíduo se dispor a contribuir cairia em 5,93 pontos percentuais. Em termos de elasticidades, uma variação em $1 \%$ no valor da DAP diminuiria em 1,44\% a probabilidade de o indivíduo se dispor a contribuir. Esse resultado representa claramente uma relação de demanda elástica; a priori, esperava-se que essa relação fosse inelástica, pois a qualidade do ar é uma característica que influencia diretamente a qualidade de vida de uma comunidade. O que pode justificar a elasticidade elástica de probabilidade de aceitação da DAP é a periodicidade das queimadas que ocorrem no período de 3 a 4 meses por ano. Caso as queimadas fossem realizadas durante todo o ano e consequentemente a poluição do ar impactasse a sociedade durante o ano inteiro, a relação entre o valor da DAP e a probabilidade de contribuir poderia ser inelástica.

O efeito marginal da renda familiar mostra que para cada variação na renda familiar de $R \$ 10,00$, a probabilidade média aumentaria em 0,12 pontos percentuais, por sua vez, uma variação de $1 \%$ na renda ocasionaria uma variação de $0,44 \%$ na probabilidade do indivíduo se dispor a contribuir para a melhoria do ar. Essa relação pode ser interpretada como uma relação renda consumo e indica que a melhoria da qualidade do ar é um bem normal.

Para os gastos com doenças respiratórias, tanto o efeito marginal quanto a elasticidade de probabilidade possuem maior expressão. Para cada aumento de $\mathrm{R} \$ 10,00$ nos gastos com doenças respiratórias, a probabilidade do indivíduo se dispor a contribuir aumentaria em 0,35 pontos percentuais, por sua vez, a elasticidade de probabilidade mostra que para uma variação de $1 \%$ nos gastos com despesas de saúde ocorreria uma variação de $0,13 \%$ na probabilidade de contribuição.

A priori, esperava-se que os entrevistados que tivessem filhos apresentassem disposição a contribuir para melhoria da qualidade do ar, fato 
esse que foi comprovado e possui impacto significativo, pois, para cada criança presente na família a probabilidade de um conjunto de resposta sim-sim aumentaria em 3,31 pontos percentuais, o que indica que a existência de crianças no lar tornaria o cidadão mais sensível à melhoria da qualidade do meio ambiente. A variável DDIST é uma variável geográfica e permitiria verificar se existe diferença entre a percepção da qualidade do ar nos dois distritos de Rio Branco, contudo, não foi significativa.

É possível verificar claramente que a DAP e a quantidade de criança exercera impacto significativo na variável dependente. Observa-se que um aumento de $\mathrm{R} \$ 4,00$ na DAP tornaria nula a probabilidade de o indivíduo se dispor a contribuir; por sua vez, uma diminuição da mesma magnitude ocasiona um aumento na probabilidade de o indivíduo se dispor a contribuir de 23,72 pontos percentuais, o que levaria a probabilidade de aceitação para 55,36\% .

Outro aspecto que merece destaque em relação à avaliação do modelo logit duplo é a eficiência na previsão. Nesse aspecto, o modelo estimado mostrou-se eficiente, pois teve um índice de acerto de aproximadamente $74 \%$. A Tabela 4 informa detalhadamente as previsões efetuadas pelo modelo.

Tabela 4 - Predições certas e erradas do modelo logit duplo pela melhoria da qualidade do ar no Estado do Acre, 2004

\begin{tabular}{cccrrrr}
\hline Respostas & Observado & $\%$ & Previsto & $\%$ & Erro & Erro \% \\
\hline SS & 67 & 27,35 & 46 & 18,78 & 21 & 31,34 \\
NN & 111 & 45,31 & 143 & 58,37 & 32 & 28,83 \\
SN & 38 & 15,51 & 31 & 12,65 & 7 & 18,42 \\
NS & 29 & 11,84 & 25 & 10,20 & 4 & 13,79 \\
Total & 245 & 100,00 & 245 & 100,00 & 64 & 26,12 \\
\hline
\end{tabular}

Fonte: Resultados da pesquisa.

Em relação às predições das escolhas dos entrevistados constatase que o modelo é bastante robusto. Observa-se que para a resposta sim-sim, teve-se um percentual de acerto de $68,66 \%$; já para ambas as respostas negativas, o nível de acerto foi de $71,17 \%$; para as respostas sim-não, teve-se um nível de acerto de $81,26 \%$; por fim, as resposta definidas por não-sim, tiveram o maior índice de acerto, de 86,21\%. 
Analisaram-se as medidas de ajustamento do logit duplo e verificaram-se as predições fornecidas pelo modelo. Agora, torna-se necessário determinar o valor monetário que representa o montante que os cidadãos estariam dispostos a pagar pela melhoria da qualidade do ar.

A disposição a pagar foi determinada através do experimento de Krinsk-Roob, com um milhão de interações, a partir do que se verificou a presença de DAPs negativas. Para contornar esse problema, foi necessário truncar o valor da DAP. Com isso, a distribuição de probabilidade da disposição a pagar deixou de ser normal. Além disso, a média da disposição a pagar aumentou bastante, pois passou de $R$ \$ 5,30 para $R$ \$ 22,70, conforme informado na Tabela 5.

Tabela 5 - Experimento de Krinsk-Roob para disposição a pagar pela melhoria da qualidade do ar no Estado do Acre, 2004

\begin{tabular}{lcc}
\hline \multicolumn{1}{c}{ Estatísticas } & DAP & DAP \\
& Probabilística & Probabilística truncada \\
\hline Média & 5,2897 & 22,6767 \\
Mediana & 5,3271 & 6,2033 \\
Máximo & 246,5078 & 246,5078 \\
Mínimo & $-221,0515$ & 0,0000 \\
Desvio-padrão & 49,4221 & 30,5125 \\
Jarque-Bera & 0,04243 & 519604,1 \\
P-valor & 0,97900 & 0,000000 \\
Simulação & 1.000 .000 & 1.000 .000 \\
\hline
\end{tabular}

Fonte: Resultado da pesquisa.

Em relação à média da disposição a pagar observou-se uma grande diferença entre as médias da DAP probabilística e da DAP probabilística truncada. Contudo, em relação à mediana, os valores foram muito próximos, e o teste de qui-quadrado não rejeitou a hipótese de que as medianas das duas séries são iguais.

A DAP probabilística apresentou valores negativos na simulação de Krinsk-Roob, indicando que alguns agentes após a implementação da despoluição do ar, terão sua utilidade diminuída.

A DAP representa o preço máximo que os agentes estão dispostos a pagar pela melhoria na qualidade do ar, contudo na economia não existe sentido para preço negativo, e conseqüentemente, torna-se necessário truncar 
a DAP probabilística para evitar preço negativo. O procedimento de truncar a DAP ocasiona dois problemas, o primeiro é a perda de informação com o truncamento da variável, e o segundo consiste em assumir a fortíssima pressuposição de que a melhoria da qualidade do ar trará benefícios a todos os agentes, ou seja, ninguém terá variações negativas na utilidade.

Utilizou-se a média ${ }^{8}$ para agregar os benefícios oriundos da despoluição do ar no Estado do Acre. Os benefícios anuais da despoluição foram estimados ${ }^{9}$ em $\mathrm{R} \$ 43.583 .455,20$, o que representa ${ }^{10}$ US\$ $17.573 .973,87$.

Determinou-se o valor dos benefícios anuais da melhoria da qualidade do ar. Surge, agora, o questionamento-base da valoração contingente, o que fazer com esse valor?

Se a sociedade está disposta a pagar aproximadamente 17,5 milhões de dólares por ano pela melhoria da qualidade do ar, esse recurso poderia ser alocado para financiar projetos de investimentos que criem condições objetivas para isso. Dado que parte da poluição do ar é exógena ao Estado do Acre, as políticas públicas no curto e médio prazos devem-se pautar nos determinantes intra-estadual da poluição do ar, ou seja, as ações e políticas públicas devem ter como objeto o produtor agrícola. Torna-se necessário a criação de mecanismos que possibilitem ao produtor rural incorporar em sua tecnologia de produção conhecimentos que permitam a ele diminuir ou acabar com a utilização das queimadas como fator de produção.

A disposição a pagar pela melhoria do ar pode ser utilizada como fonte de recurso para financiar projetos de extensão rural que leve ao produtor uma tecnologia "limpa" de produção agropecuária. Nesse sentido, a tecno-

${ }^{8}$ Hanemann (1984 e 1989) considerou que a mediana é a alternativa mais adequada para a estimativa dos benefícios, pois ela é reconhecida como uma medida de tendência central de uma distribuição, ou seja, não é tão sensível a inclusão de outliers. Contudo, JOHANSON et al. (1989) salientaram que, em termos de agregação, a média é a medida de tendência central; logo, mesmo na presença de outliers, a média é a medida mais adequada para agregação. Vale destacar que o conceito implícito de mediana é $50 \%$ a favor e $50 \%$ contra, o que não leva a alocações eficientes no sentido de Pareto.

${ }^{9} \mathrm{O}$ cálculo dos benefícios é efetuado da seguinte maneira: multiplica-se o valor da média (da DAP truncada) por 12 (meses do ano) e pelo total de famílias estimado do Estado do Acre, que no caso foi de 159.998 em 2004.

${ }^{10}$ A cotação utilizada para conversão foi a do dia 05 de maio de 2005 , em que US\$ 1,00 $=\mathrm{R} \$ 2,48$. 
logia pode ser conduzida no tocante a criar formas alternativas de preparo do solo, bem como no financiamento de atividades correlacionadas com agronegócio que possuam menores impactos sobre o meio ambiente.

Outro aspecto que merece ser ressaltado reside na análise benefício/ custo da melhoria do ar, no ano de 2004. O SUS efetuou um gasto ${ }^{11}$ de $\mathrm{R} \$ 2.067 .180,00$, aproximadamente US\$ 833,540.00 com despesas relativas a morbidades respiratórias. Uma simples análise beneficio/custo indica que para cada dólar de gastos com morbidades respiratórias, a melhoria do ar acarreta um benefício de US\$21.08.

Em termos de avaliação de projeto, um coeficiente B/C de 21,08 mostra que a melhoria da qualidade ambiental é um projeto de investimento viável. Outro indicador tradicionalmente utilizado na avaliação de projeto, o valor presente líquido (VPL) indica que os benefícios da despoluição para um período de 10 anos, utilizando-se uma taxa de $6 \%$ a.a., é de US\$129,345,977.53, através da Tabela 6 visualiza-se os indicadores econômicos da melhoria da qualidade do ar.

Tabela 6 - Indicadores econômicos pela melhoria na qualidade do ar no Estado do Acre, 2004

\begin{tabular}{lrr}
\hline \multicolumn{1}{c}{ Indicadores } & \multicolumn{1}{c}{$\mathbf{R} \mathbf{U}$} & US $\mathbf{}$ \\
\hline DAP mensal familiar & 22,70 & 9.15 \\
DAP anual & 272,40 & 109,83 \\
Benefício anual & $43.583 .455,20$ & $17,573,973.87$ \\
Benefício em 10 anos & $320.778 .024,27$ & $129,345,977.53$ \\
Gastos com internações por morbidades & $2.067 .180,57$ & $833,540.33$ \\
respiratórias em 2004 & 21,08 & 21,08 \\
B/C
\end{tabular}

Fonte: Resultados da pesquisa.

Uma forma alternativa de visualização da viabilidade econômica da melhoria da qualidade do ar no Estado do Acre é visualizada na Tabela 7, que mostra a sensibilidade do B/C sujeito às variações no valor da DAP,

${ }^{11}$ Evidentemente, que nem todo gasto com doenças respiratórias é em função das queimadas, contundo, para essa análise, o valor considerado será o valor total dos gastos do SUS, a implicação disso está na diminuição dos benefícios, no entanto, isso não chega a ser prejudicial para as análises, em função dos grandes benefícios que a melhoria da qualidade do ar traz a sociedade conforme mostra o B/C. 
sendo válido ressaltar que o valor de $\mathrm{R} \$ 22,70$ corresponde à máxima disposição a pagar pela melhoria da qualidade ambiental.

O valor da DAP por familiar que torna indiferente entre prover ou não o projeto de investimento de melhoria na qualidade do ar corresponde a 4,74\% da DAP máxima, o que representa R \$ 1,08, ou seja, algo em torno de R\$ 0,27 por membro da família.

Tabela 7 - Análise de sensibilidade de indicadores selecionados pela melhoria da qualidade do ar no Estado do Acre, 2004

\begin{tabular}{crrcrc}
\hline $\begin{array}{c}\text { \% do valor } \\
\text { máximo da DAP } \\
\text { familiar mensal }\end{array}$ & $\begin{array}{c}\text { Benefícios } \\
\text { anuais em R\$ } \$\end{array}$ & $\begin{array}{c}\text { DAP mensal } \\
\text { por família } \\
\text { em R\$ }\end{array}$ & $\begin{array}{c}\text { DAP mensal } \\
\text { por família } \\
\text { em US\$ }\end{array}$ & B/C & $\begin{array}{c}\text { DAP mensal em } \\
\text { R por membro } \\
\text { da família* }\end{array}$ \\
\hline 1,00 & $435.834,55$ & 0,23 & 0.09 & 0,21 & 0,06 \\
5,00 & $2.179 .172,76$ & 1,14 & 0.46 & 1,05 & 0,28 \\
10,00 & $4.358 .345,52$ & 2,27 & 0.91 & 2,11 & 0,57 \\
25,00 & $10.895 .863,80$ & 5,68 & 2.29 & 5,27 & 1,42 \\
50,00 & $21.791 .727,60$ & 11,35 & 4.57 & 10,54 & 2,84 \\
75,00 & $32.687 .591,40$ & 17,03 & 6.86 & 15,81 & 4,26 \\
100,00 & $43.583 .455,20$ & 22,70 & 9.15 & 21,08 & 5,68 \\
\hline
\end{tabular}

Fonte: Resultados da pesquisa.

* Considerando quatro membros por família.

Por fim, o valor da despoluição do ar pode ser utilizado como um parâmetro para determinação de multas punitivas para os agentes que poluem o ar. Assim, determinando-se quais os emissores e quanto emitiram é possível judicialmente multá-los pelo menos no montante que a sociedade se dispõe a contribuir pela melhoria da qualidade do ar.

\section{Conclusão}

As queimadas causam malefícios à sociedade, principalmente à saúde dos cidadãos. Nesse contexto, a mensuração da disposição a pagar pela melhoria da qualidade do ar é uma informação relevante para os diversos níveis de tomada de decisão para formulação de políticas públicas que visem criar mecanismos para a diminuição dos impactos negativos ocasionados pela utilização do fogo no processo produtivo da agropecuária da região amazônica, e principalmente do Estado do Acre. 
De forma geral, esta pesquisa buscou determinar a disposição a pagar pela melhoria da qualidade do ar no Acre. Especificamente, pretendeu-se, verificar quais variáveis sócio-econômicas possuem maior relevância para explicar a decisão dos indivíduos aceitarem ou não a DAP e comparar a DAP agregada com o custo total das morbidades respiratórias ocasionados pelas queimadas no ano de 2004 .

O modelo estimado apresentou bons indicadores de ajustamento. Além disso, mostrou que as variáveis com maior impacto sobre a probabilidade média do indivíduo em se dispor a contribuir pela melhoria da qualidade do ar no Estado do Acre foram, em ordem crescente: o valor da disposição a pagar, a quantidade de crianças presentes na família e o montante dos gastos realizados com morbidades respiratórias.

Os resultados mostraram de forma clara que a melhoria da qualidade do ar no Acre é um projeto de investimento viável, visto que para cada dólar investido na melhoria da qualidade do ar os benefícios estimados são de aproximadamente US\$21,00.

A principal recomendação deste estudo é relacionada com a utilização do uso do solo para a produção agropecuária na Amazônia. É preciso que os órgãos governamentais nas mais diversas instâncias, e as organizações não-governamentais criem condições objetivas para o desenvolvimento de tecnologias alternativas ao uso do fogo na agropecuária, e, em um segundo momento, essa tecnologia deve ser difundida ao produtor agropecuário através dos serviços técnicos e de extensão rural.

\section{Referências bibliográficas}

CAMERON, T.A. A new paradigm for valuing non-market goods using referendum data: maximum likelihood estimation by censored logistic regression. Journal of Environmental Economics and Management, n. 15, p. 355-379, 1988.

CAMERON, T.A.; GUIGGIN, J. Estimation using contingent valuation data from a "dichotomus choice with follow-up" questionnaire. Journal of Environmental Economics and Management, n. 27, p. 218-234, 1994.

CARSON, R.T.; HANEMANN, W.M.; MITCHELL, R.C. Determining the 
demand for public goods by simulating referendums at different tax price. San Diego: Manuscript, University of California, 1986.

COOPER, J.C.; HANEMMANN, W.M.; SIGNORELLO, G. One-and-onehalf-bound dichotomous choice contingent valuation. The Reviews of Economics and Statistics, v. 84, n. 4, p. 742-750, Nov. 2002.

HANEMANN, M., LOOMIS, J., KANNINEN, B. Statistical efficiency of double-bounded dichotomous choice contingent valuation. American Journal of Agricultural Economics, n. 71, p. 1255-1263, 1991.

HANEMANN, M.W. Welfare evaluation in contingent valuation experiments with discrete responses. American Journal of Agricultural Economics, n. 66, p. 332-341, 1984.

JOHANSON, B.D.K.; MALER, K.G. Welfare evaluation in contingent valuation experiments with discrete responses: comment. American Journal of Agricultural Economics, v. 71, p. 1054-1056, 1989.

KRINSKY, I., ROBB, A.L. On approximating the statistical properties of elasticities. The reviews of economics and statistics. V. 68, p. 715 - 719, 1986.

PEARCE, D.W., TURNER, R.K. Economics of natural resources and environment. Londres: Harvester Wheatshealf, 1990. 178 p.

SILVA, R.G. Disposição a pagar para evitar danos à saúde oriundos das queimadas: uma aplicação do método de valoração contingente no Estado do Acre. 121 p. Tese (Doutorado em Economia Aplicada) - Universidade Federal de Viçosa, Viçosa, MG, 2005.

Recebido em outubro de 2005 e revisto em abril de 2006 